\title{
Software Phase Correction Technique for Passive Radar
}

\author{
Paulo AC Marques \\ ISEL-Instituto de Telecomunicações, R. Conselheiro Emídio Navarro 1, Lisbon, Portugal
}

\begin{tabular}{l} 
ARTICLE INFO \\
\hline Article history's: \\
Received 09 December 2020 \\
Revised 10 December 2020 \\
Accepted 29 December 2020 \\
\end{tabular}

\section{Keywords:}

Passive Radar,

Phase Correction,

Low Cost Hardware,

Target of Opportunity,

Multistatic Radar.

\begin{abstract}
A traditional radar actively transmits pulses and receive the corresponding echoes. By computing the time taken to receive the echoes the system is able to detect targets and estimate their ranges. However, mainly for military applications, an active radar has a major drawback: it can be detected by the enemy since it radiates electromagnetic pulses. As such, there is currently high research and development activity in the field of passive radar systems. In this alternative scenario, the radar does transmit any signal. Instead, it uses signals already present in the environment, such as TV, radio broadcasts and satellite digital video broadcast as transmitters of opportunity. By measuring the time difference between the signal received by the transmitter of opportunity and the signal echoed by the targets, the radar can detect targets and estimate their ranges. In scenarios which use very low power illuminators of opportunity, such as satellite DVB-S or GPS, relatively long integration times are required in order to obtain reasonable values of signal to noise ratio. This implies that, besides frequency correction, the coherency of local oscillators in the receivers need to be maintained during long time intervals, which can be very difficult. The paper presents a novel phase correction technique for passive radar which uses targets of opportunity, already present in the target area, as references to maintain the coherency of the oscillators for all the integration interval. The proposed methodology is quite simple and enables the use of low-cost hardware with independent oscillators for the reference and surveillance channels which can be geographically distributed. The obtained results illustrate the effectiveness and applicability of the method.
\end{abstract}

This work is licensed under a Creative Commons Attribution-Share Alike 4.0

Paulo AC Marques,

ISEL-Instituto de Telecomunicações, R. Conselheiro Emídio Navarro 1, Lisbon, Portugal

Email: pmarques@isel.pt.

\section{INTRODUCTION}

Radar is a system that uses radio frequency (RF) signals to detect objects, estimate their trajectory and, in more advanced systems, perform imaging. A radar system has several advantages over optical systems such as being able to penetrate clouds and obtain information independently of being day or night, among others.

An active radar transmits RF signals and records the corresponding echoes that are processed in order to obtain information about the target area. This kind of system has a major drawback, mainly for military applications: it can be detected by the enemy since it transmits relatively high-power signals.

A very interesting alternative is the passive radar. In this scenario, the radar is completely silent and does not transmit any signal. It uses the transmitters already present in the target area, the so-called transmitters of opportunity. An additional advantage is that a passive radar does not occupy an already crowded RF spectrum, which, mainly for civilian applications, is very important.

Research in the field of passive radar nowadays is very intense due to their application on both civilian and military context [1-5] [6-12]. Since passive radar can work in a passive and covert mode, by exploiting the transmitters of opportunity available in the scenario of operation, it is particularly interesting for Defense applications. 
Let us consider the geometry for passive radar presented in Figure 1. For the sake of simplicity, a single transmitter of opportunity is available in this example. The radar acquires two versions of the signal radiated by the transmitter of opportunity: one version is the direct signal acquired by the reference channel; the other version - the signal scattered by the target - is acquired by the surveillance channel. The signals are then ready to be down converted and digitized. The cross-correlation can then be computed to detect the targets of interest.

In a monostatic configuration, i.e., both receiving antennas are collocated, both channels can share the same local oscillator and the time delay at which the correlation has peaks, determines the target ranges. In a bistatic configuration, i.e., both antennas are separated by a distance comparable to the expected target distance, a system for synchronizing the reference and surveillance channels is mandatory since the local oscillator cannot be the shared between channels.

Passive radar systems have been implemented with success in the scenarios that use ground-based transmitters, with high power, such as broadcast FM radio, analog and terrestrial digital video broadcast (DVBT) $[13,14,15,16]$. A completely different challenge arises if space-based transmitters of opportunity such as satellite digital video broadcast satellites (DVB-S) or GPS emitters are used. In this situation the signals arrive to Earth surface with very low power and the target detection becomes more difficult $[17,18,19]$.

One way of overcoming the limitation of employing very low power illuminators of opportunity consists in use relatively long integration times in order to obtain reasonable values of signal to noise ratio (SNR) [17, $18,20,21]$. However, the usage of long integration times has the major drawback of demanding frequency correction and maintaining coherence between the receiving local oscillators (LO), which can be very hard.

A common way of maintaining the coherency between the LO in the receivers resides in the usage of a single local oscillator which signal is fed to all the receiving channels [17]. The obvious limitation is the relatively short distance at which receiving channels must be between each other.

Such a system is illustrated in Figure 2, which presents a typical low-cost two channel passive coherent receiver built with hardware that is easily available. The reference and surveillance radio frequency signals are received by two antennas and fed to a two-channel analog receiver which down converts both to baseband. The baseband signals are then converted from the analog domain to the digital domain by a digital oscilloscope which transfers them to a conventional PC for further processing.

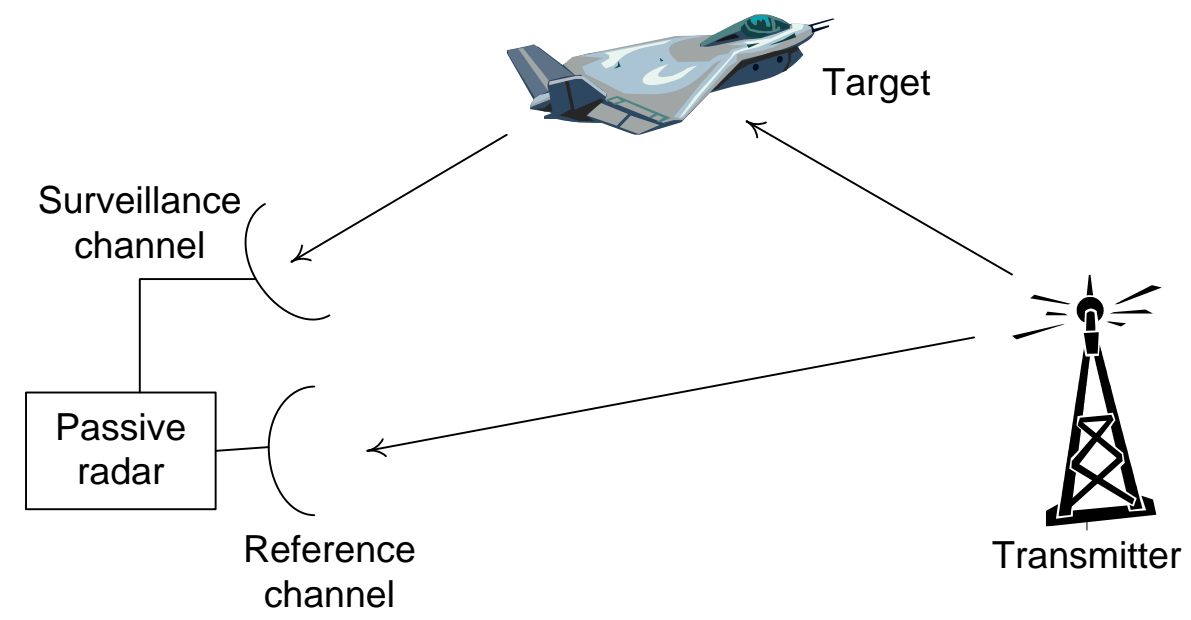

Fig. 1. Passive radar uses signals already present in the target area. The radar receives the direct signal in the reference channel and the reflected signal in the surveillance channel. With proper processing the target can be detected.

Another simple and effective method of synchronization consists in using a physical link between both channels' local oscillators, as depicted in Figure 3. In this method the local oscillators of both reference and surveillance channels are the same, at each of the reception stages, i.e., radio frequency (RF) to intermediate frequency (IF) and from IF to base band (BB).

A big disadvantage of the method is the limitation on the distance between the receivers can be placed, since they need to be physically linked. In some scenarios this limitation cannot be observed, such as in some multistatic configurations demanding geographically spread channels [11] [17]. In this situation, more sophisticated and expensive methods are required, such as those based on GPS [18][22].

This article presents an alternative method to maintain coherency among the local oscillators of the surveillance and reference channels. The basic idea consists in using static and high relatively high reflectivity 
targets that are present in the region of interest to use as reference for phase correction. The method elaborates on works [19] and [21] from the same author. The presented solution does not demand a physical link between the local oscillators which can be crucial in geometries where the receivers need to be far apart from each other's, such as in multi-static configurations [8].

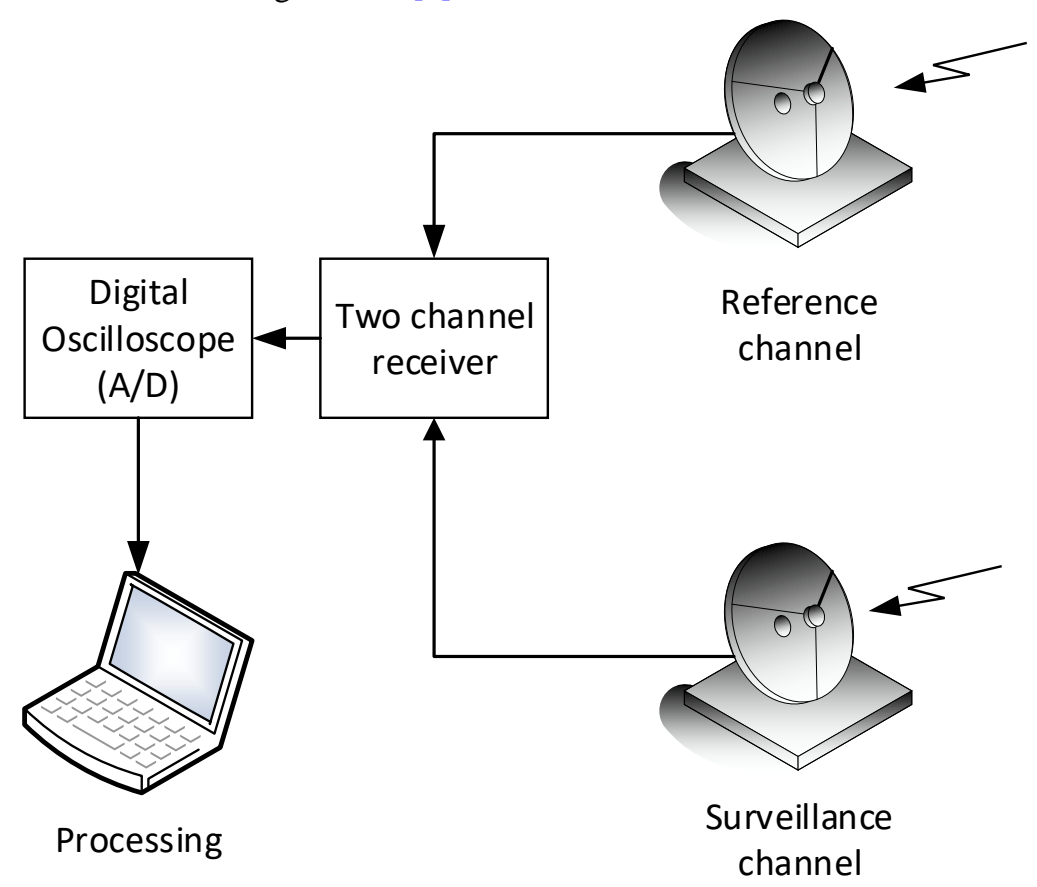

Fig. 2. Passive radar can be implemented with low cost hardware commonly accessible in electronics labs.

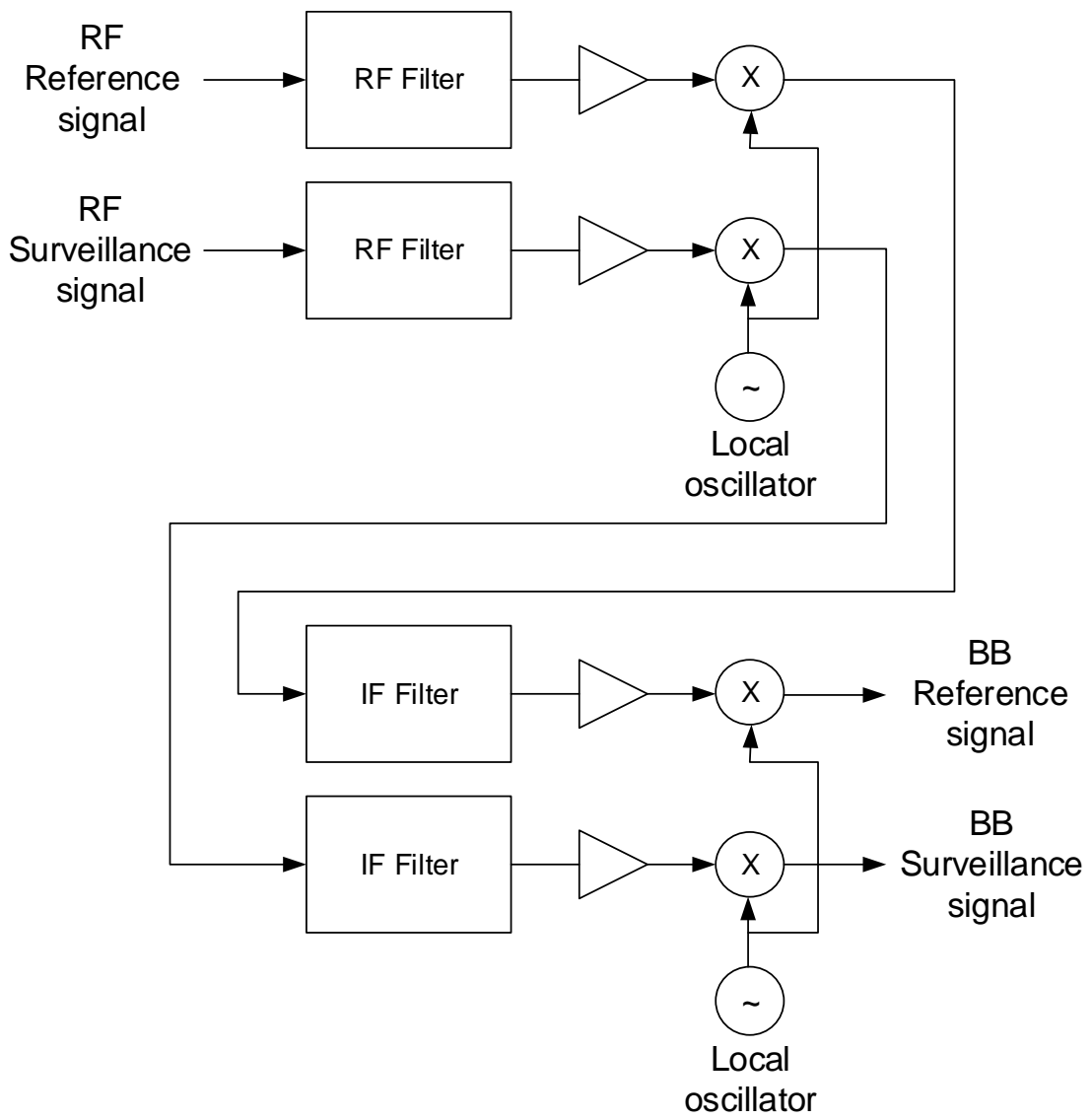

Fig. 3. Synchronization of surveillance and reference channels using a physical link for the local oscillators. 
The paper is organized as follows. Section 2 presents the proposed methodology. Section 3 presents results illustrating the effectiveness of the methodology. Section 4 draws the conclusions and discusses future work.

\section{THEORY}

Target detection in a passive radar is mainly dependent on the cross-correlation between the surveillance signal, $s_{s}(t)$, and the reference signal, $s_{r}(t)$.

In order to produce a range-velocity map, the correlation is made with several shifted replicas of the reference signal [14]:

$$
C(\tau, v)=\left|\int_{-\infty}^{+\infty} s_{r}(t) s_{s}^{*}(t-\tau) \exp \left(j 2 \pi f_{d} t\right) d t\right|
$$

where $\tau$ is related with the range $r$ by $\tau=2 r c, v$ is the target velocity projected in the line of sight (LOS) of the receiver, $f_{d}=2 v / \lambda$ is the Doppler frequency shift and $\lambda$ is the signal wavelength.

In low signal-to-clutter ratio (SCR) scenarios, the objects may not be detectable without some prior signal processing. This is particularly true if the system uses satellite illuminators of opportunity.

In this low SCR scenarios, the typical approach uses N measurements of the same target area that are coherently added to increase detectability [17] [18]:

$$
s_{r_{s}}(t)=\sum_{n=0}^{N-1} s_{r_{n}}(t)
$$

where $s_{r_{s}}$ is received signal resulting from the coherent addition of the $\mathrm{N}$ measurements on the reference signal. This way, there is an increase in the SCR proportional to the number of measurements $\mathrm{N}$.

This strategy, however, requires frequency and phase coherence for all the receiving channels. If one of the conditions is not met, the signals will not be summed coherently, and the SCR will not be increased.

In a scenario where the receiver for each channel is relatively distant from the remaining channels, each receiver needs to have its independent local oscillator. Since the oscillators become physically independent, if no phase correction is made, the detection capability is seriously impaired.

\section{PROPOSED METHODOLOGY}

Let us consider that a certain target is present in all the measurements, at a known position. In such a case, we could use it as reference to correct the LO phase, after pulse compression for each measurement. This way the signals could be summed coherently, increasing the SCR. Figure 4 illustrates the considered scenario.

The chief idea is to use as reference a man-made structure with relatively high radar cross section (RCS) that is present in the target area, as shown in Figure 4. Since the location of this structure is known, we can impose a phase after pulse compression, for all measurements. This way, the phase drift that would otherwise occur between the LO on the reference and surveillance channels, as well as with the surveillance channel itself for different times, can be corrected.

Therefore, instead of directly summing several signals from coherent channels, a phase correction step is performed. The correction is made by using a reference target which produces echoes in the surveillance channel. In order to facilitate the correction, the compensation must be done after compression of each pulse, taking advantage of the SNR gain due to the pulse compression.

Let us consider that the opportunity transmitter generates signal $s_{o}(t)$. The reference channel receives signal $s_{r}(t)=s_{o}\left(t-t_{0}\right)$, where $t_{0}$ is the delay due to the distance between the transmitter of opportunity and the receiving antenna on the reference channel; $t_{0}$ includes other delays due to the hardware at the receiving reference channel.

For the sake of simplicity, let us assume the presence of a reference object with RCS $\sigma_{r_{o}}$ and, additionally, consider the presence of single unknown target with RCS $\sigma_{t}$. 


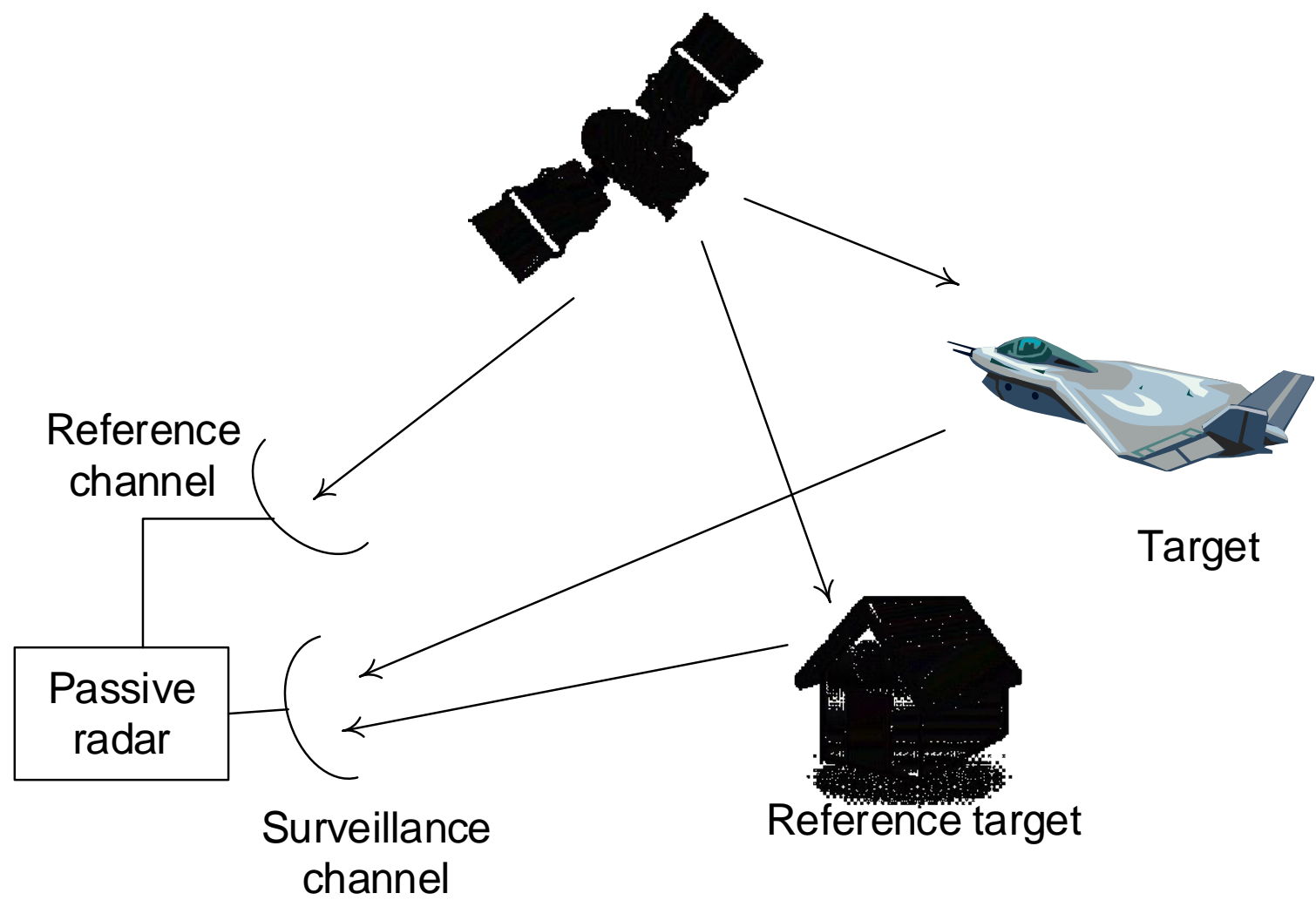

Fig. 4. A stationary object with high RCS can be used as reference target to correct the phase of the local oscillators and maintain their coherency during the acquisition interval.

For each measurement, the signal at the surveillance channel will be given by

$$
s_{s}(t)=s_{r_{o}}(t)+s_{t}(t)=\sigma_{r_{o}} s_{o}\left(t-t_{r_{o}}\right)+\sigma_{t} s_{t}\left(t-t_{t}\right)
$$

where $t_{r o}$ is the delay of the signal due to the reference object and $t_{t}$ is the delay due to the unknown and weaker target.

The algorithm consists then in making $N$ readings for the reference channel and surveillance channel, thus obtaining, for the $i$ th reading:

$$
\begin{gathered}
s_{s}(t)=s_{r_{o}}(t)+s_{t}(t)=\sigma_{r_{o}} s_{o}\left(t-t_{r_{o}}\right)+\sigma_{t} s_{t}\left(t-t_{t}\right) \\
s_{r i}(t)=s_{o i}(t)
\end{gathered}
$$

the $i$ th reference signal and

$$
s_{s i}(t)=\left[s_{\text {roi }}(t)+s_{t i}(t)\right] e^{j \theta_{i}}
$$

is the ith reading of surveillance signal, where each reading can have a different phase $\theta_{i}$ due to the natural drift of the local oscillator used in the surveillance channel.

For each iteration both signals are correlated, obtaining $C_{i}(\tau)$. The position of the reference target is taken as the maximum of the $t$ th correlation:

$$
\tau_{r i}=\max \left[\operatorname{abs}\left(C_{i}(\tau)\right)\right]
$$

Then, by obtaining the phase $\phi_{i}=\arg C_{i}\left(\tau_{r i}\right)$ on the maximum position, it can be corrected by imposing null phase and, therefore, summing it coherently:

$$
C(t)=C(\tau)+C_{i}\left(\tau-\tau_{c}\right) e^{-\phi_{i}}
$$

\section{RESULTS}

This section presents results illustrating the effectiveness of the proposed strategy for phase correction by using a target of opportunity. Table 1 presents the simulated scenario where five targets are present, their ranges and relative RCS. The first target is used as reference for the phase correction. 
Table 1. Target ranges and corresponding relative RCS

\begin{tabular}{ccc}
\hline Target & Range $[\mathrm{m}]$ & RCS \\
\hline 1 (reference) & 128 & 1 \\
$\mathbf{2}$ & 256 & 0.5 \\
$\mathbf{3}$ & 512 & 0.25 \\
$\mathbf{4}$ & 756 & 0.125 \\
$\mathbf{5}$ & 1024 & 0.0625 \\
\hline
\end{tabular}

The simulation considers both channels with independent and completely unsynchronized local oscillators, i. e., the phase changes randomly for each measurement, and uniformly, in the $2 \pi$ interval. The number of measurements is $\mathrm{N}=1024$.

For illustration purposes only, let us now consider the situation where the phase of the local oscillators is not corrected. Figure 5 illustrates the resulting signal for $\mathrm{SCR}=3 \mathrm{~dB}$, after averaging 1024 measurements, without phase correction. Due to the phase drift between measurements the resulting signal is incoherently combined and, as such, none of the targets is visible. This way, the obtained results are clearly inadequate for target detection.

In order to better understand the results that can be expected with the proposed algorithm for phase correction, Figure 6 presents Monte-Carlo run for four scenarios with SCR ranging from -6dB to $3 \mathrm{~dB}$. As can be seen, if the reference target has SCR better than $3 \mathrm{~dB}$ the target range and reflectivity estimation converges to an accuracy high enough for target detection.

The previous prediction is confirmed in Figure 7 that illustrates the detection results, for $\mathrm{SCR}=3 \mathrm{~dB}$, after $\mathrm{N}=1024$ iterations. The first target, at range $256 \mathrm{~m}$, was used as reference for phase correction. As can be seen, targets 1 to 4 become clearly visible at ranges $128 \mathrm{~m}, 256 \mathrm{~m}, 512 \mathrm{~m}$ and $756 \mathrm{~m}$. Only target 5 , located at range $1024 \mathrm{~m}$, is not distinguishable from the clutter due to its comparatively low RCS. The estimation results can be used for target detection and for target RCS estimation.

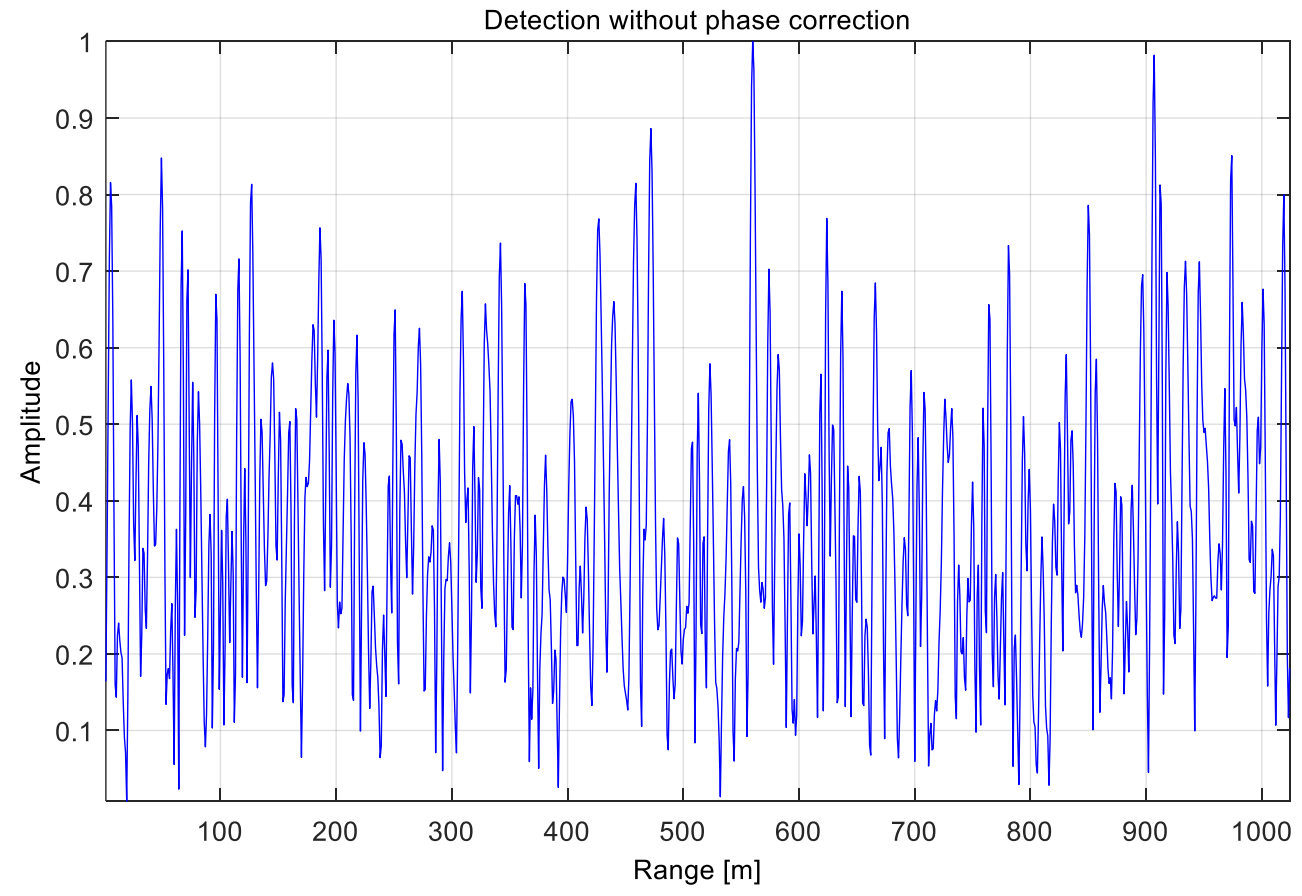

Fig. 5. Without phase correction, none of the targets can be detected. 


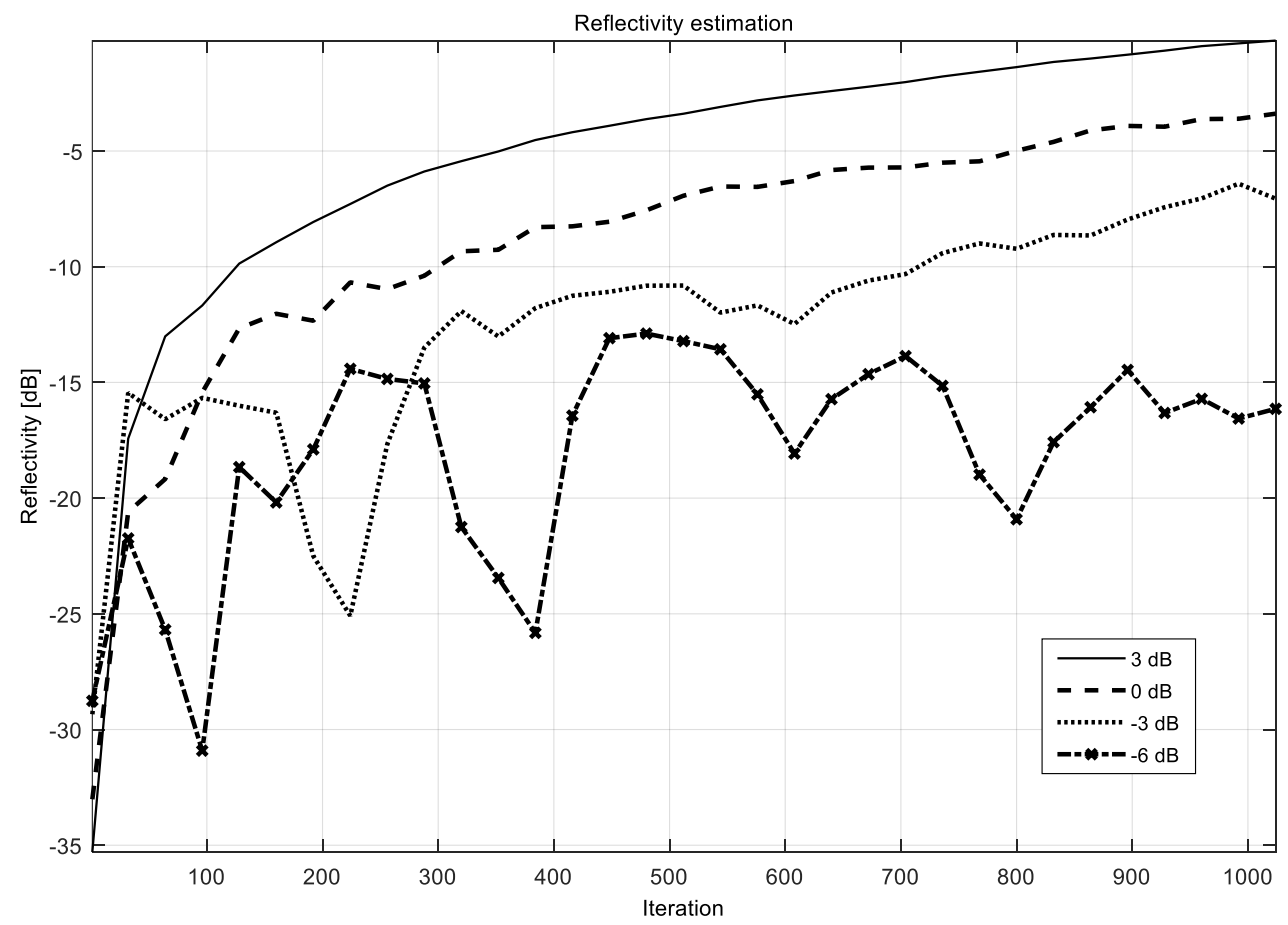

Fig. 6. Target reflectivity estimation as function of the signal-to-clutter ratio of the reference target used for phase correction of the local oscillators.

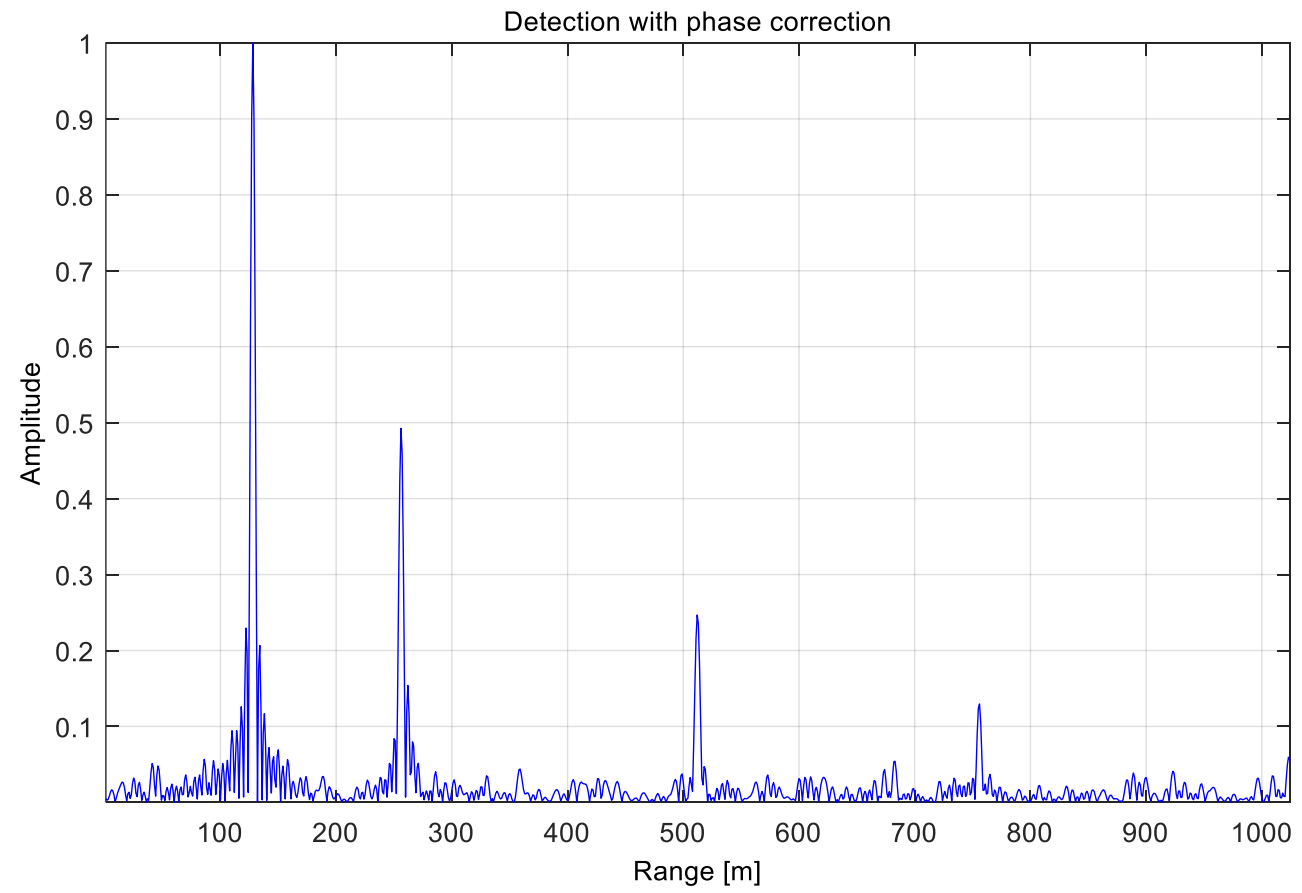

Fig. 7. Using a reference target for phase correction, all the targets present in the target area become clearly detectable after signal cross-correlation.

Table 2 presents average target relative RCS error estimate as function of SCR after 256 Monte Carlo experiments. Four scenarios are considered for SRC ranging from $-3 \mathrm{~dB}$ to $6 \mathrm{~dB}$. 
As expected, the average error decreases for reference targets with higher SCR. Although the error may be unacceptable for a reference target with $\mathrm{SCR} \leq 0 \mathrm{~dB}$, the same is not true for $\mathrm{SCR} \geq 3 \mathrm{~dB}$. Here, the average RCS error estimate is $5.4 \%$ and $1.3 \%$ for $\mathrm{SCR}=3 \mathrm{~dB}$ and $\mathrm{SRC}=6 \mathrm{~dB}$, respectively, which is perfectly acceptable for many operational scenarios.

Table 2. Average error estimates for the relative SCR

\begin{tabular}{cc}
\hline SCR [dB] & Error avg $(\%)$ \\
\hline $\mathbf{6}$ & 1.3 \\
$\mathbf{3}$ & 5.4 \\
$\mathbf{0}$ & 55 \\
$\mathbf{- 3}$ & 77 \\
\hline
\end{tabular}

From the obtained results, we can expect that the technique herein proposed will provide good results for phase correction, target detection and, eventually, imaging, if the SCR for the reference target is, at least, $3 \mathrm{~dB}$.

\section{CONCLUSION}

Passive radar systems are very interesting mainly for military operations since they do not transmit any signal being therefore very hard to detect by the enemy. This kind of system uses signals already present in the target area, such as TV, radio broadcasts or satellite such as DVB-S and GPS.

In the situation where very low power transmitters of opportunity are used, relatively long integration times are required to obtain acceptable values of signal to clutter ratio. Long integration times, however, demand coherency between local oscillators for an equal length of time, which can be very difficult and expensive.

This paper presented a low-cost, all-software solution to obtain coherency between the signals acquired by the passive radar. The novel phase correction technique uses targets of opportunity, known to be present in the target area, as references.

The obtained results show that the technique can be used if the target used as reference for phase correction exhibits a SCR which is, at least, $3 \mathrm{~dB}$.

When compared with alternative costly solutions such as those based on GPS atomic clocks for synchronization of the local oscillators, the proposed technique can be used on very low-cost hardware, with completely independent oscillators on each channel. Additionally, the phase correction algorithm is computationally light and simple.

The presented solution does not demand a physical link between the local oscillators which can be crucial in geometries where the receivers need to be far apart from each other's, such as in multi-static passive radar configurations.

Future work includes the evaluation of the technique limits and its application to moving targets detection and, eventually, imaging.

\section{REFERENCES}

[1] M. A. Nuhoglu, Y. K. Alp and F. C. Akyon, "Deep Learning for Radar Signal Detection in Electronic Warfare Systems," 2020 IEEE Radar Conference (RadarConf20), Florence, Italy, 2020, pp. 1-6, DOI: https://doi.org/10.1109/RadarConf2043947.2020.9266381

[2] Z. He, Y. Yang and W. Chen, "A Hybrid integration method for moving target detection with GNSS-based passive radar," in IEEE Journal of Selected Topics in Applied Earth Observations and Remote Sensing, DOI: https://doi.org/10.1109/JSTARS.2020.3037200

[3] F. Colone, "DVB-T based Passive Forward Scatter Radar: inherent limitations and enabling solutions," in IEEE Transactions on Aerospace and Electronic Systems, DOI: https://doi.org/10.1109/TAES.2020.3035435

[4] S. Mercier, S. Bidon, D. Roque and C. Enderli, "Comparison of Correlation-Based OFDM Radar Receivers," in IEEE Transactions on Aerospace and Electronic Systems, vol. 56, no. 6, pp. 4796-4813, Dec. 2020, DOI: https://doi.org/10.1109/TAES.2020.3003704

[5] P. Wojaczek, F. Colone, D. Cristallini and P. Lombardo, "Reciprocal-Filter-Based STAP for Passive Radar on Moving Platforms," in IEEE Transactions on Aerospace and Electronic Systems, vol. 55, no. 2, pp. 967-988, April 2019, DOI: https://doi.org/10.1109/TAES.2018.2867688

[6] M. H. Javidan, A. Zaimbashi and J. Liu, "Target Detection in Passive Radar Under Noisy Reference Channel: A New Threshold-Setting Strategy," in IEEE Transactions on Aerospace and Electronic Systems, vol. 56, no. 6, pp. 4711 4722, Dec. 2020, DOI: https://doi.org/10.1109/TAES.2020.2999998

[7] Y. Fang et al., "Improved Passive SAR Imaging With DVB-T Transmissions," in IEEE Transactions on Geoscience and Remote Sensing, vol. 58, no. 7, pp. 5066-5076, July 2020, DOI: https://doi.org/10.1109/TGRS.2020.2972156 
[8] A. D. Byrd, R. D. Palmer and C. J. Fulton, "Development of a Low-Cost Multistatic Passive Weather Radar Network," in IEEE Transactions on Geoscience and Remote Sensing, vol. 58, no. 4, pp. 2796-2808, April 2020, DOI: https://doi.org/10.1109/TGRS.2019.2955606

[9] J. L. Garry and G. E. Smith, "Experimental Observations of Micro-Doppler Signatures With Passive Radar," in IEEE Transactions on Aerospace and Electronic Systems, vol. 55, no. 2, pp. 1045-1052, April 2019, DOI: https://doi.org/10.1109/TAES.2019.2895584

[10] S. Paine, D. W. O'Hagan, M. Inggs, C. Schüpbach and U. Böniger, "Evaluating the Performance of FM-Based PCL Radar in the Presence of Jamming," in IEEE Transactions on Aerospace and Electronic Systems, vol. 55, no. 2, pp. 631-643, April 2019, DOI: https://doi.org/10.1109/TAES.2018.2858158

[11] A. R. Persico, P. Kirkland, C. Clemente, J. J. Soraghan and M. Vasile, "CubeSat-Based Passive Bistatic Radar for Space Situational Awareness: A Feasibility Study," in IEEE Transactions on Aerospace and Electronic Systems, vol. 55, no. 1, pp. 476-485, Feb. 2019, DOI: https://doi.org/10.1109/TAES.2018.2848340

[12] H. D. Griffiths, A. J. Garnett, C. J. Baker and S. Keaveney, "Bistatic radar using satellite-borne illuminators of opportunity," 92 International Conference on Radar, Brighton, UK, 1992, pp. 276-279.

[13] H. Griffiths, "Passive Bistatic Radar and Waveform Diversity", Cranfield University, Defense College of Management and Technology, Shrivenham, RTO-EN-SET-119, 2009.

[14] D. Olivadese, E. Giusti, D. Petri, M. Martorella, A. Capria and F. Berizzi, "Passive ISAR With DVB-T Signals," in IEEE Transactions on Geoscience and Remote Sensing, vol. 51, no. 8, pp. 4508-4517, Aug. 2013, DOI: https://doi.org/10.1109/TGRS.2012.2236339

[15] P. E. Howland, D. Maksimiuk and G. Reitsma, "FM radio based bistatic radar," in IEE Proceedings - Radar, Sonar and Navigation, vol. 152, no. 3, pp. 107-115, 3 June 2005, DOI: https://doi.org/10.1049/ip-rsn:20045077

[16] F. Colone, P. Falcone, C. Bongioanni and P. Lombardo, "WiFi-Based Passive Bistatic Radar: Data Processing Schemes and Experimental Results," in IEEE Transactions on Aerospace and Electronic Systems, vol. 48, no. 2, pp. 1061-1079, APRIL 2012, DOI: https://doi.org/10.1109/TAES.2012.6178049

[17] S. Tholert, and D. Hounam, "A Passive Multistatic CW Radar using Geostationary Illuminators", European Conference on Synthetic Aperture Radar, Dresden, Germany, May, 2006.

[18] D. Cristallini et al., "Space-based passive radar enabled by the new generation of geostationary broadcast satellites," 2010 IEEE Aerospace Conference, Big Sky, MT, 2010, pp. 1-11, DOI: https://doi.org/10.1109/AERO.2010.5446694

[19] P. Marques, "Opportunity Targets as References for Phase Correction on Passive Radar Channels", International Radar Symposium, Dresden, Germany, Vol. 1, pp. 1-4, June, 2015. DOI: https://doi.org/10.1109/IRS.2015.7226298

[20] P. Marques, Challenges and Practical Applications of Passive Radar - Keynote Speech, NATO Science and Technology Organization NATO Specialist Meeting on Multi-band Multi-mode Rada SET-231, Lisbon, Portugal, Vol. 1, pp. 1 - 4, October, 2016.

[21] P. Marques, "Phase Correction for Passive Radar Using Targets of Opportunity", IEEE International Geoscience and Remote Sensing Symposium, Quebec, Canada, pp. 717-720, July, $2014 . \quad$ DOI: https://doi.org/10.1109/IGARSS.2014.6946524

[22] K. Kulpa, M. Malanowski, "From Klein Heidelberg to Modern Multistatic Passive Radar", International Radar Symposium, Ulm, Germany, pp. 1-9, 2019. DOI: https://doi.org/10.23919/IRS.2019.8768176

\section{BIOGRAPHY OF AUTHORS}

Paulo Marques, received the B.S. degree in electronics and telecommunications engineering from Higher Engineering Institute of Lisbon (ISEL) in 1990 and the E. E., M.Sc. and Ph.D. degrees in electrical and computer engineering from Technical University of Lisbon (IST) in 1993, 1997, and 2004, respectively. In his $\mathrm{PhD}$ thesis he addressed the problem of detection of moving targets and full velocity vector and trajectory estimation using single channel SAR. In the past years he has been contributing in a wide range of problems in synthetic aperture radar, noise radar and passive radar in several European projects and in NATO research groups. Paulo Marques is author and co-author of more than 70 papers in which he is mostly first author and frequently serves as a referee for many international journals and conferences. He is currently a Coordinator Professor at ISEL, Department of Electronics and Communications Engineering and is also a researcher with the Pattern and Image Analysis group at Telecommunications Institute of Lisbon. His expertise topics include Synthetic Aperture Radar, Moving Target Indication, Signal and Image Processing, Noise Radar and Passive Radar. 